\title{
Necrotizing Sarcoid Granulomatosis With an Uncommon Manifestation: Clinicopathological Features and Review of Literature
}

\author{
Chiara Giraudo MD, Nazarena Nannini MD, Elisabetta Balestro MD, Alessia Meneghin MD, \\ Francesca Lunardi PhD, Roberta Polverosi MD, and Fiorella Calabrese MD
}

\begin{abstract}
We report a rare case of an incidental diagnosis of necrotizing sarcoid granulomatosis (NSG) in a 60-y-old non-smoking male. The patient was admitted to the hospital for sudden back pain. Chest $\mathrm{x}$-ray revealed areas of parenchymal consolidation and high-resolution computed tomography demonstrated a pulmonary nodular pattern with no lymph node enlargement. All laboratory and pulmonary function tests were normal. Bronchoscopy with bronchoalveolar lavage showed no sign of infection or specific inflammation. The diagnosis of NSG was made by histopathological examination of a surgical lung biopsy and by excluding other causes of granulomatous disease. In paucisymptomatic/asymptomatic patients, as in our case, therapy is not necessary, with a good prognosis and complete recovery. NSG is a rare systemic disease similar to sarcoidosis and Wegener's granulomatosis with a benign clinical course and should always be considered for patients with nodular pulmonary lesions even with subclinical or uncommon features. Key words: back pain; pulmonary nodules; granulomatous disease; histopathology; sarcoidosis; Wegener's granulomatosis. [Respir Care 2014;59(9):e132-e136. @ 2014 Daedalus Enterprises]
\end{abstract}

\section{Introduction}

Necrotizing sarcoid granulomatosis (NSG) is an uncommon systemic disease of unknown etiology and incidence. NSG is more common in females than males, and most patients are smokers. The age of presentation varies, with distribution of cases between the second and sixth decades of life. ${ }^{1}$ It was described for the first time in 1973 by Liebow ${ }^{2}$ as a lung granulomatous disease characterized by sarcoid-like

\footnotetext{
Drs Giraudo and Polverosi are affiliated with the Department of Radiology, Venetian Oncology Institute, Padua, Italy. Drs Nannini, Balestro, Lunardi, and Calabrese are affiliated with Department of Cardiac, Thoracic and Vascular Sciences, University of Padua, Padua, Italy. Dr Meneghin is affiliated with the Department of Medicine, Hospital of Montebelluna, Treviso, Italy.
}

The authors have disclosed no conflicts of interest.

Correspondence: Fiorella Calabrese MD, Department of Cardiac, Thoracic and Vascular Sciences, Section of Pathological Anatomy, University of Padua, Via Gabelli 61, 35121 Padua, Italy. E-mail: fiorella. calabrese@unipd.it.

DOI: $10.4187 /$ respcare. 02842 granulomas, vasculitis, and a variable degree of necrosis. Since then, several case reports have documented the existence of NSG, mainly reporting lung involvement. ${ }^{1,3-10}$ The etiology and pathogenesis are still unknown.

It has not yet been determined whether this disease is a specific entity, a variant of sarcoidosis or pulmonary vasculitis (Churg-Strauss syndrome or Wegener's granulomatosis), or a hypersensitivity reaction, as some features are found in all these diseases. NSG is diagnosed on the basis of morphological features and shares common histological and clinical patterns with sarcoidosis.

The radiological pattern consists of multiple bilateral nodules and infiltrates or appears as a solitary mass. Evidence of cavitation and hilar lymphadenopathy is occasionally seen. The clinical presentation can be extremely variable: cough is the most common manifestation, followed by chest pain, dyspnea, weight loss, and fatigue. Subclinical or uncommon manifestations, as in our case, have rarely been described.1,3,4

We report a case of NSG in which the final diagnosis was based on an accurate radiological and overall morphological approach. A brief review and update of the literature are also provided, focusing mainly on lung NSG. 


\section{Case Report}

A 60 -y-old white non-smoking male presented at the hospital with sudden back pain (eased with paracetamol). At admission, he did not report any other symptoms, such as fever or malaise, or any kind of trauma, and a medical history was taken to rule out ingested or injected factors. Physical examination was quite unremarkable except for reduced breath sounds at the right lung base. Chest x-ray was performed and showed bilateral lower lobe areas of consolidations associated with linear atelectasis. High-resolution computed tomography was performed, and multiple bilateral nodular lesions were detected, localized mostly in the lower lobes ( $>2 \mathrm{~cm}$ maximum diameter), with a prevalent subpleural distribution (Fig. 1). Some lesions had the halo sign, but no cavitations were detected. An abdominal ultrasound was normal.

Laboratory analysis revealed a low number of white blood cells $(3,760$ cells $/ \mu \mathrm{L})$ and lymphocytes $(720$ cells $/ \mu \mathrm{L}$ ), especially of the CD4 subset. Erythrocyte sedimentation rate, C-reactive protein, electrophoresis of proteins, angiotensin-converting enzyme concentration, serum immunoglobulins, and autoantibodies (anti-nuclear antibodies, anti-extractable nuclear antigen antibodies, anti-neutrophil cytoplasmic antibodies) were normal. Microbiological testing for human immunodeficiency virus (blood), QuantiFERON-TB Gold (blood), Aspergillus fumigatus (bronchoalveolar lavage), Histoplasma capsulatum (urine and blood), and Chlamydia (bronchoalveolar lavage and blood) were negative. Pulmonary function tests revealed normal lung volume, with a mild $(74 \%)$ reduction in diffusing capacity of the lung for carbon monoxide.

Bronchoscopy with bronchoalveolar lavage fluid was negative for infections and malignant cells. The differential cell count was normal (CD4/CD8 ratio of 1.8\%). A transbronchial biopsy was carried out, but it did not provide any diagnostic information; thus, a thoracoscopic lung biopsy was performed.

Histological analysis showed a severe, mostly inflammatory architectural rearrangement (Fig. 2). The inflammatory infiltrate was characterized mainly by aggregates of epithelioid granulomas with surrounding chronic inflammation. Granulomas were located primarily in subpleural areas and along bronchovascular bundles. Many granulomas showed a confluent pattern with extensive central areas of necrosis. Transmural lymphocytic vasculitis was also seen. Gomori methenamine silver, Giemsa, Gram, Warthin-Starry, and periodic acid-Schiff staining did not reveal any bacteria or fungi. Atypical and typical mycobacteria (investigated also by nested polymerase chain reaction ${ }^{11}$ ) were negative. No foreign body was identified by polarized light microscopy.

Histological analysis led to a final diagnosis of NSG. Without any therapy, the patient's symptoms disappeared

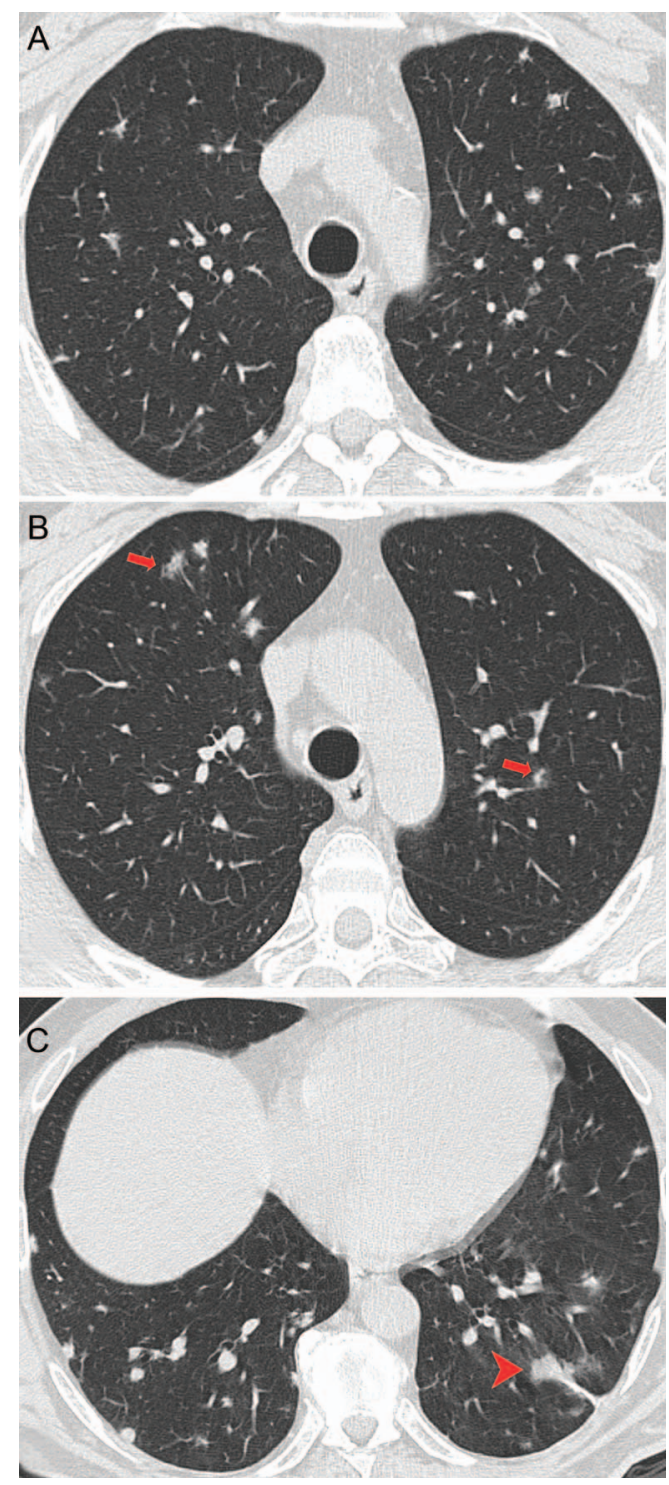

Fig. 1. A: High-resolution computed tomography shows multiple bilateral nodular lesions localized mostly in the lower lobes with a prevalent subpleural distribution. B: Some lesions have the halo sign (arrows). C: In the left lower lobe, a larger nodule is adherent to the left costovertebral space (arrowhead).

within 4 weeks following the biopsy. Serial clinical and radiological follow-ups were scheduled, and chest x-ray 1 month after the surgery showed a significant reduction in the remaining pulmonary infiltrates. To date, there is no evidence of progression of the lesions.

\section{Discussion}

Since the first report by Liebow, ${ }^{2}$ several authors have described the existence of NSG, ${ }^{1,3-10,12-21}$ mostly involving lung parenchyma (Table 1). The etiology and pathogenesis 

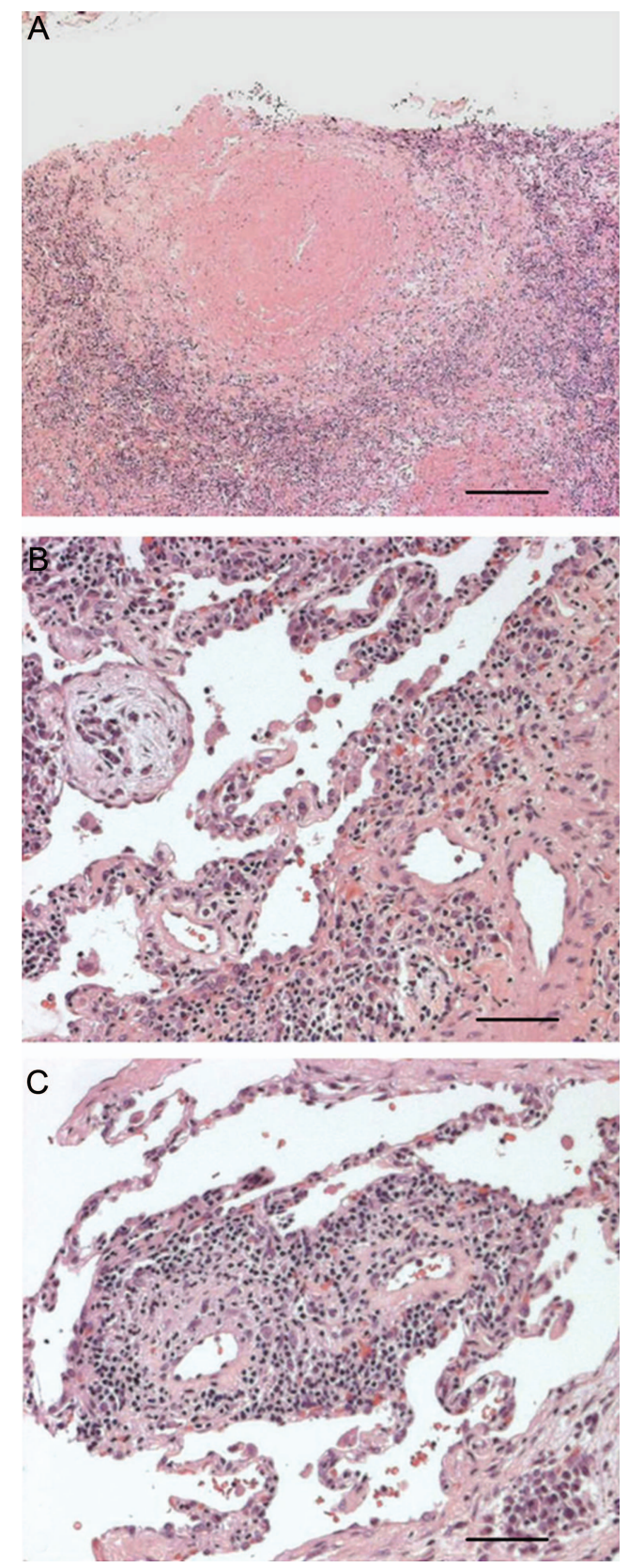

Fig. 2. A: Granuloma with a large central area of parenchymal necrosis with a coagulative pattern. Scale bar $=500 \mu \mathrm{m}$. B: Foci of organizing pneumonia. Scale bar $=150 \mu \mathrm{m}$. C: Lymphocytic vasculitis involving small arteries. Scale bar $=150 \mu \mathrm{m}$.

are unknown, and a biopsy specimen is required for final diagnosis.

The clinical presentation is quite variable and nonspecific. Cough is the most common clinical manifestation of NSG, followed by pleural chest pain, dyspnea, fever, and constitutional symptoms of weight loss. ${ }^{6,18}$ However, $\sim 25 \%$ of patients are paucisymptomatic/asymptomatic. ${ }^{20}$ Limited information is available in the literature regarding laboratory tests. The most important clinicoradiological differential diagnoses of NSG are Wegener's granulomatosis, rheumatoid arthritis, sarcoidosis, and metastasis. It is important to distinguish NSG from systemic vasculitis or metastasis because of differences in prognosis and response to treatment.

Unlike patients with Wegener's granulomatosis, patients with NSG have no upper airway disease, nephritis, or systemic vasculitis. Moreover, the round opacity typically demonstrates thick patchy wall cavitation areas, ${ }^{22,23}$ which were completely absent in our case. Uveitis or cutaneous lesions, involvement of hilar lymph nodes, and increased angiotensin-converting enzyme levels, which are usually frequent in sarcoidosis, are rare in NSG, as in our case.

Although NSG and sarcoidosis seem to have different presentations, there is still debate about whether the 2 diseases are distinct entities. ${ }^{18}$ Indeed, there are some similarities between sarcoidosis and NSG. Both diseases have a favorable prognosis, a similar immune mechanism, and distribution of granulomas. ${ }^{24}$ Furthermore, $5 \%$ of patients with sarcoidosis show the typical histology of NSG. ${ }^{25,26}$

High-resolution computed tomography of rheumatoid lung nodules shows well defined nodules with lobulated margins. The size of isolated nodular lesions may increase during the acute phase of the disease proportional to the antibody titer. The hypothesis of rheumatoid lung disease was excluded in our case because all antibody titers were negative, and no arthralgias were reported in the clinical history.

In our case, the nodules were mainly peripheral and localized mostly in the lower lobes; thus, lung metastasis also had to be considered. Metastatic opacities usually have regular and well-defined margins but sometimes are hazy with a halo sign, as in choriocarcinomas and angiosarcomas. Cavitations or calcifications depend on the histological pattern of the primary tumor. We excluded metastatic disease because no primary tumor had been detected, and the patient was in a relatively stable condition.

Biohumoral analysis and high-resolution computed tomography can be used when NSG is suspected; however, lung biopsy is the cornerstone for establishing the diagnosis of NSG. This is particularly true in cases with subclinical or uncommon presentation, as in our patient. Indeed, back pain could be due to degenerative discopathy (spondyloarthrosis) or subpleural lower lobe localization of nodules (pleuritic chest pain).

From a pathological point of view, the most important differential diagnoses are Wegener's granulomatosis and infection. Well-formed non-necrotizing sarcoid granulomas are rarely seen in Wegener's granulomatosis. Moreover, the vasculitis of Wegener's granulomatosis is more necrotizing and often suppurative. ${ }^{18,19,27}$ A differential diagnosis with infection is more difficult: conventional microbiological analysis and use of overall tissue ancillary techniques such as special stains, immunohistochemistry, 
Table 1. Lung Necrotizing Sarcoid Granulomatosis: Literature Review

\begin{tabular}{|c|c|c|c|c|c|c|c|c|c|c|}
\hline Reference & $\begin{array}{c}\text { Cases } \\
(n)\end{array}$ & Sex & $\begin{array}{l}\text { Cough } \\
(n)\end{array}$ & $\begin{array}{c}\text { Fever } \\
(n)\end{array}$ & $\begin{array}{l}\text { Dyspnea } \\
(n)\end{array}$ & $\begin{array}{l}\text { Chest Pain } \\
\text { (n) }\end{array}$ & $\begin{array}{c}\text { Night Sweats } \\
(n)\end{array}$ & $\begin{array}{l}\text { Pleuritic Pain } \\
(n)\end{array}$ & $\begin{array}{c}\text { Other } \\
(n)^{*}\end{array}$ & $\begin{array}{c}\text { No Treatment } \\
(n)\end{array}$ \\
\hline Churg et $\mathrm{al}^{3}$ & 12 & $10 \mathrm{~F} / 2 \mathrm{M}$ & 6 & 3 & 3 & & & 1 & & 3 \\
\hline Beach et $\mathrm{al}^{12}$ & 1 & M & 1 & & & & & & 1 & 0 \\
\hline Koss et $\mathrm{al}^{4}$ & 13 & $10 \mathrm{~F} / 3 \mathrm{M}$ & 5 & 4 & 3 & 8 & 2 & & 4 & 2 \\
\hline Singh et al ${ }^{13}$ & 1 & F & & & & & & & 1 & 0 \\
\hline Rolfes et $\mathrm{al}^{5}$ & 1 & $\mathrm{~F}$ & 1 & 1 & & & & & & 0 \\
\hline Chittock et al ${ }^{6}$ & 7 & $5 \mathrm{~F} / 2 \mathrm{M}$ & 7 & 7 & 7 & & 7 & 6 & & 1 \\
\hline Niimi et $\mathrm{al}^{14}$ & 3 & $1 \mathrm{~F} / 2 \mathrm{M}$ & NR & NR & NR & NR & NR & NR & NR & NR \\
\hline Hsu et al ${ }^{15}$ & 16 & NR & NR & NR & NR & NR & NR & NR & NR & NR \\
\hline Le Gall et al ${ }^{16}$ & 3 & $1 \mathrm{~F} / 2 \mathrm{M}$ & 3 & & & & & & 2 & 0 \\
\hline Dykhuizen et al ${ }^{17}$ & 1 & F & & 1 & & & & & 2 & 0 \\
\hline Tauber et al ${ }^{7} \mathrm{E} / 1999$ & 1 & $\mathrm{~F}$ & & & & 1 & & & 1 & NR \\
\hline Heinrich et $\mathrm{al}^{8}$ & 1 & F & & & & & & & 2 & 1 \\
\hline Popper et al ${ }^{18}$ & 10 & $5 \mathrm{~F} / 5 \mathrm{M}$ & NR & NR & NR & NR & NR & NR & NR & NR \\
\hline Lazzarini et al ${ }^{19}$ & 4 & F & 2 & & & & & 2 & 4 & 3 \\
\hline Quaden et $\mathrm{al}^{1}$ & 14 & $10 \mathrm{~F} / 4 \mathrm{M}$ & 5 & 5 & 4 & 4 & 2 & & 2 & 5 \\
\hline Arfi et $\mathrm{al}^{20}$ & 2 & F & & & 1 & & & & 1 & 2 \\
\hline Epping et $\mathrm{al}^{21}$ & 1 & M & 1 & & 1 & 1 & & & 2 & 0 \\
\hline Panigada et $\mathrm{al}^{9}$ & 1 & M & 1 & 1 & & & & 1 & 1 & 0 \\
\hline Sahin et al ${ }^{10}$ & 1 & M & 1 & & & & & & 1 & 0 \\
\hline Total, $n(\%)$ & $93 \dagger$ & $24 \mathrm{M} / 53 \mathrm{~F}$ & $33(52)$ & $22(34)$ & $19(30)$ & $14(22)$ & $11(17)$ & $10(16)$ & $24(38)$ & $17(27)$ \\
\hline \multicolumn{11}{|c|}{$\begin{array}{l}\text { * This includes shoulder pain, subcutaneous nodules, sore throat, conjunctivitis, headache, VI-VII left cranial nerve palsy, diarrhea, lower limb weakness, neurological symptoms, weight loss, } \\
\text { shortness of breath, and malaise. } \\
\dagger \text { Symptoms were reported in } 64 \text { of } 93 \text { cases; thus, different symptom percentages were calculated in } 64 \text { patients. Treatment information was available in } 63 \text { patients; thus, no treatment percentage } \\
\text { was calculated in } 63 \text { cases. } \\
\mathrm{F}=\text { female } \\
\mathrm{M}=\text { male } \\
\mathrm{NR}=\text { not reported }\end{array}$} \\
\hline
\end{tabular}

and molecular analysis (the latter for mycobacterial detection) are important. Above all, the use of a highly specific and sensitive molecular technique such as nested polymerase chain reaction for mycobacterial detection is mandatory. This is particularly true in cases with subclinical or uncommon features, as in the case presented here.

The prognosis of patients with NSG has been described as favorable. Indeed, the clinical course is generally benign, with a good response to steroid treatment or without the need for therapy. ${ }^{1,6,17}$ The known case reports/series on lung NSG are listed in Table 1. In many cases, no medical treatment was required, as in our case. Treatment seems to be associated with relapse and should be started only when symptoms are severe.

We have presented a case of NSG with an uncommon clinical manifestation. The final diagnosis was based on a multidisciplinary approach and overall on accurate morphological and molecular analysis. Our case supports the fact that the disease has a benign clinical course with complete spontaneous recovery especially when tissue lesions are not extensive and when there are no constitutional symptoms.

\section{ACKNOWLEDGMENTS}

We thank Ms Judith Wilson (Department of Cardiac, Thoracic and Vascular Sciences, University of Padua, Padua, Italy) for the English revision of this manuscript.

\section{REFERENCES}

1. Quaden C, Tillie-Leblond I, Delobbe A, Delaunois L, Verstraeten A, Demedts M, Wallaert B. Necrotising sarcoid granulomatosis: clinical, functional, endoscopical and radiographical evaluations. Eur Resp J 2005;26(5):778-785.

2. Liebow AA. The J. Burns Amberson lecture-pulmonary angiitis and granulomatosis. Am Rev Respir Dis 1973;108(1):1-18.

3. Churg A, Carrington CB, Gupta R. Necrotizing sarcoid granulomatosis. Chest 1979;76(4):406-413.

4. Koss MN, Hochholzer L, Feigin DS, Garancis JC, Ward PA. Necrotizing sarcoid-like granulomatosis: clinical, pathologic and immunopathologic findings. Hum Pathol 1980;11(5 Suppl):510-519.

5. Rolfes DB, Weiss MA, Sanders MA. Necrotizing sarcoid granulomatosis with suppurative features. Am J Clin Pathol 1984;82(5):602-607.

6. Chittock DR, Joseph MG, Paterson NA, McFadden RG. Necrotizing sarcoid granulomatosis with pleural involvement. Clinical and radiographic features. Chest 1994;106(3):672-676.

7. Tauber E, Wojnarowski C, Horcher E, Dekan G, Frischer T. Necrotizing sarcoid granulomatosis in a 14-yr-old female. Eur Resp J 1999;13(3):703-705. 


\section{Uncommon Manifestation of Necrotizing Sarcoid Granulomatosis}

8. Heinrich D, Gordjani N, Trusen A, Marx A, Hebestreit H. Necrotizing sarcoid granulomatosis: a rarity in childhood. Pediatr Pulmonol 2003;35(5):407-411.

9. Panigada S, Ullmann N, Sacco O, Gambini C, Bush A, Rossi GA. Necrotizing sarcoid granulomatosis of the lung in a 12-year-old boy with an atypical clinical course. Pediatr Pulmonol 2012;47(8):831835.

10. Sahin H, Ceylan N, Bayraktaroglu S, Tasbakan S, Veral A, Savas R. Necrotizing sarcoid granulomatosis mimicking lung malignancy: MDCT, PET-CT and pathologic findings. Iran J Radiol 2012;9(1):37-41.

11. Azov AG, Koch J, Hamilton-Dutoit SJ. Improved diagnosis of mycobacterial infections in formalin-fixed and paraffin-embedded sections with nested polymerase chain reaction. APMIS 2005;113(9): 586-593.

12. Beach RC, Corrin B, Scopes JW, Graham E. Necrotizing sarcoid granulomatosis with neurologic lesions in a child. J Pediatr 1980; 97(6):950-953.

13. Singh N, Cole S, Krause PJ, Conway M, Garcia L. Necrotizing sarcoid granulomatosis with extrapulmonary involvement. Clinical, pathologic, ultrastructural, and immunologic features. Am Rev Respir Dis 1981;124(2):189-192.

14. Niimi H, Hartman TE, Müller NL. Necrotizing sarcoid granulomatosis: computed tomography and pathologic findings. J Comput Assist Tomogr 1995;19(6):920-923.

15. Hsu RM, Connors AF Jr, Tomashefski JF Jr. Histologic, microbiologic and clinical correlates of the diagnosis of sarcoidosis by transbronchial biopsy. Arch Pathol Lab Med 1996;120(4):364-368.

16. Le Gall F, Loeuillet L, Delaval P, Thoreux PH, Desrues B, Ramée P. Necrotizing sarcoid granulomatosis with and without extrapulmonary involvement. Pathol Res Pract 1996;192(3):306-313; discussion 314.
17. Dykhuizen RS, Smith CC, Kennedy MM, McLay KA, Cockburn JS, Kerr KM. Necrotizing sarcoid granulomatosis with extrapulmonary involvement. Eur Respir J 1997;10(1):245-247.

18. Popper HH, Klemen H, Colby TV, Churg A. Necrotizing sarcoid granulomatosis-is it different from nodular sarcoidosis? Pneumologie 2003;57(5):268-271

19. Lazzarini LC, de Fatima do Amparo Teixeira M, Souza Rodrigues R, Marcos Nunes Valiante P. Necrotizing sarcoid granulomatosis in a family of patients with sarcoidosis reinforces the association between both entities. Respiration 2008;76(3):356-360.

20. Arfi J, Kerrou K, Traore S, Huchet V, Bolly A, Antoine M, et al. F-18 FDG PET/CT findings in pulmonary necrotizing sarcoid granulomatosis. Clin Nucl Med 2010;35(9):697-700.

21. Epping G, Schwengle CA, Aliredjo RP, Wagenaar M. Necrotizing sarcoid granulomatosis: a case report and review of progresses in this disease. Health 2011;3(8):534-536.

22. MartinezF, Chung JH, Digumarthy SR, Kanne JP, Abbott GF, Shepard JA, et al. Common and uncommon manifestations of Wegener granulomatosis at chest CT: radiologic-pathologic correlation. Radiographics 2012;32(1):51-69.

23. Chew HC, Chan YM, Issam AJ, Koh MS. A patient with hearing loss, mediastinal lymphadenopathy, and cavitatory pulmonary nodules. Chest 2010;138(6):1500-1504.

24. Spiteri MA, Gledhill A, Campbell D, Clarke SW. Necrotizing sarcoid granulomatosis. Br J Dis Chest 1987;81(1):70-75.

25. Churg A. Pulmonary angiitis and granulomatosis revisited. Hum Pathol 1983;14(10):868-883

26. Rosen Y, Moon S, Huang CT, Gourin A, Lyons HA. Granulomatous pulmonary angiitis in sarcoidosis. Arch Pathol Lab Med 1977;101(4): 170-174.

27. Ma Y, Gal A, Koss MN. The pathology of pulmonary sarcoidosis: update. Semin Diagn Pathol 2007;24(3):150-161. 